\title{
Issues with RFID usage in ubiquitous computing applications
}

\author{
Christian Floerkemeier and Matthias Lampe \\ Institute for Pervasive Computing \\ Department of Computer Science \\ ETH Zurich, Switzerland \\ \{floerkem|lampe\}@inf.ethz.ch
}

\begin{abstract}
Radio Frequency Identification (RFID) has recently received a lot of attention as an augmentation technology in the ubiquitous computing domain. In this paper we present various sources of error in passive RFID systems, which can make the reliable operation of RFID augmented applications a challenge. To illustrate these sources of error, we equipped playing cards with RFID tags and measured the performance of the RFID system during the different stages of a typical card game. The paper also shows how appropriate system design can help to deal with the imperfections associated with RFID.
\end{abstract}

\section{Introduction}

In his famous article Mark Weiser describes a vision of ubiquitous computing in which technology is seamlessly integrated into the environment and provides useful services to humans in their everyday lives [8]. The potential of Radio Frequency Identification (RFID) tags to contribute to the realization of this vision has been demonstrated by many researchers over the years. Examples include prototypes such as the Magic Medicine Cabinet by Wan [6], the augmentation of desktop items by Want et al. [7] and smart shelves by Decker et al. [1]. These prototypes show that RFID technology has many benefits over other identification technologies because it does not require line-of-sight alignment, multiple tags can be identified almost simultaneously, and the tags do not destroy the integrity or aesthetics of the original object. Due to the low cost of passive RFID tags and the fact that they operate without a battery, there are, however, also some weaknesses associated with RFID-based object identification. Particularly in a multi-tag and multi-reader configuration, the phenomenon of false negative reads occurs, where a tag that is present is not detected.

The goal of this paper is to illustrate the problem of failed RFID reads with the help of a sample application, identify potential causes of the failed reads, and suggest ways to deal with the resulting uncertainty from an application and system perspective. In particular, we address the problems of collisions on the air interface and tag detuning.

The following section gives an introduction to RFID system components and Section 3 looks at the various causes of false negative reads. Section 4 suggests 
ways to deal with the uncertainty caused by failed RFID reads from an application and system design perspective. Section 5 provides a conclusion.

\section{RFID Primer}

RFID systems consist of two main components: the RFID tag, which is attached to the object to be identified and serves as the data carrier, and the RFID reader, which can read from and sometimes also write data to the tag. Tags typically consist of a microchip that stores data and a coupling element, such as a coiled antenna, used to communicate via radio frequency communication. The readers usually consist of a radio frequency module, a control unit, and a coupling element to interrogate the tags via radio frequency communication. There is a wide variety of RFID systems available. The reader is referred to the book by Finkenzeller [2] for an in-depth classification of RFID systems.

The experiments presented in this paper use the Philips I-CODE System ${ }^{1}$, which operates at $13.56 \mathrm{MHz}$ and which is based on the ISO 15693 standard for RFID. The I-CODE tags obtain their power from the magnetic field generated by the reader through inductive coupling. The magnetic field induces a current in the coupling element of the smart label, which provides the microchip with power. The inductively coupled RFID system consequently behaves much like loosely coupled transformers. The ISO 15693 protocol employs a variant of slotted Aloha for access to the shared communication medium, known as framed Aloha [5].

\section{Failed RFID tag reads and their causes}

Failure to detect tags that are present in the read range of a reader can be due to a variety of causes including collisions on the air interface, tag detuning, tag misalignment, and metal and water in the vicinity of the RFID system. To illustrate the failed tag reads caused by some of these phenomena we equipped playing cards with RFID tags similar to prior work by Römer [3]. Although other scenarios could have been used to demonstrate some of the challenges involved in the use of RFID, we believe that the playing card scenario is an appropriate example because it aptly demonstrates the most common causes of failed tag reads. The pictures in Figure 1 show the RFID tags on the back of the playing cards with the RFID antenna of the I-CODE System in the background.

To illustrate the different causes of false negative reads, we carried out measurements in a number of configurations that typically occur during a card game. It is evident from Figure 1 and Figure 2 that on average we do not detect all of the 10 tags present in a single frame in any of the arrangements considered and that the arrangement of the cards with respect to the reader and with respect to each other has a strong influence on the read performance. In the following subsections we address the individual causes of the failed reads.

\footnotetext{
$\overline{{ }^{1} \text { http://www.semiconductors.philips.com/markets/identification/products/icode/ }}$
} 


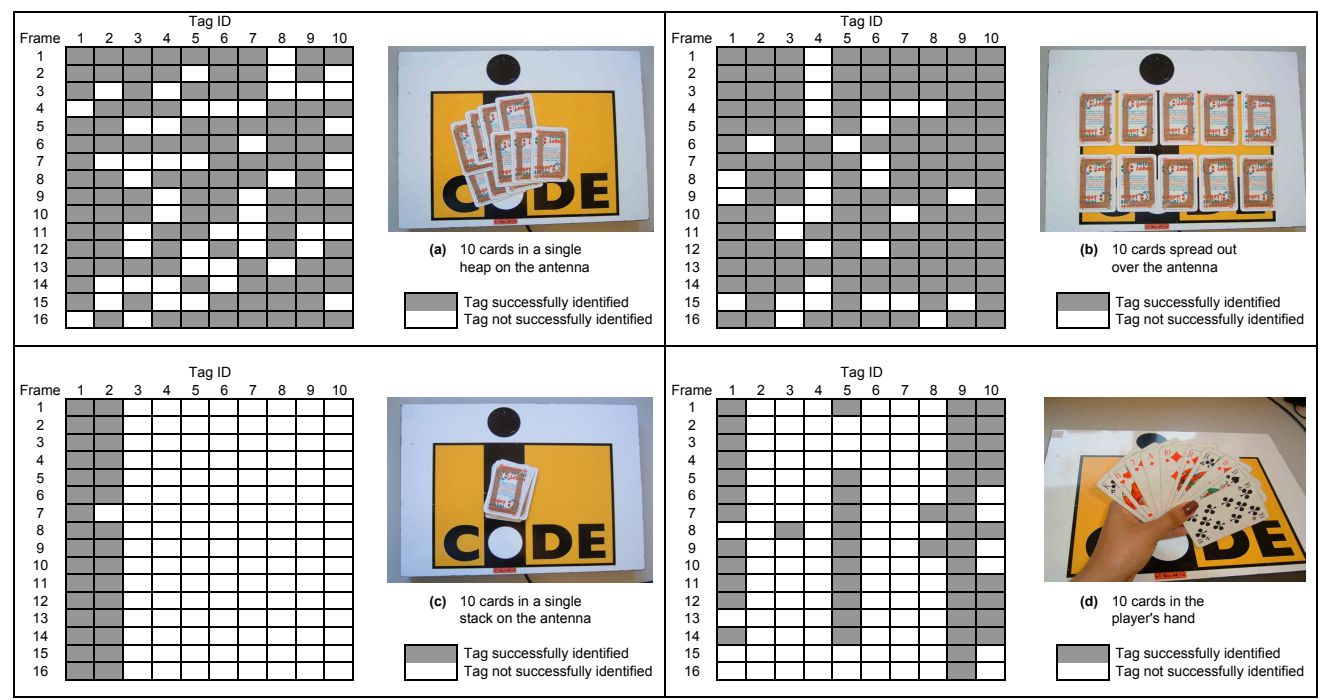

Fig. 1. Four different arrangements of 10 playing cards equipped with RFID tags: (a) in a heap on the antenna, (b) spread out over the antenna, (c) stacked on top of each other, and (d) in the player's hands. The patterns to the left of the images show a snapshot of the data captured by the reader. A dark field indicates a successful detection of a tag in a frame; a light field indicates a failed detection. These measurements were carried out with 32 time slots per frame and a frame rate of $5 \mathrm{~Hz}$.

\subsection{Tag collisions}

As mentioned earlier, the system used for our tests employs an anti-collision algorithm based on framed Aloha. In most circumstances, tags transmitting their ID in the same time slot cannot be detected. Exceptions to this rule are due to the capture effect [9], where the reader manages to detect the data sent by one of the tags correctly, although multiple tags respond in the same time slot.

In a stochastic anti-collision algorithm there is hence always a chance that a tag is not detected for at least the duration of a single frame, if more than a single tag is present. Obviously, the probability of collisions decreases with the number of available time slots (see Figure 2) and increases with the number of electronic tags present. Wieselthier et al. [9] developed expressions for the probability of successful transmission in framed Aloha with no capture. We used their analysis to calculate the expected number of tags detected per frame (see bars labelled "prediction" in Figure 2). The slight discrepancy between the prediction and the observed data for the two scenarios that perform well is believed to be due to the capture effect, which is not considered in our estimate. The relatively good match between the prediction and the observation indicates that all negative reads are due to collisions on the air interface in these cases. 


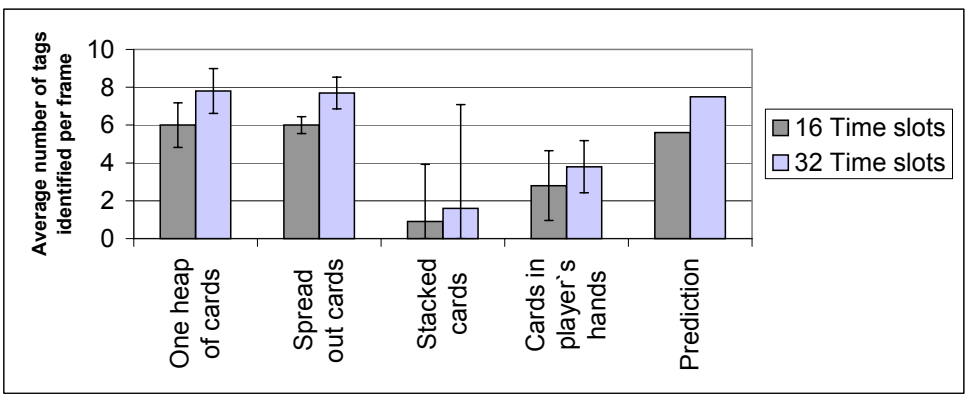

Fig. 2. Average number of tags identified per frame vs. the different card arrangement for frames with 16 and 32 time slots. The prediction bars represent the expected number of tags successfully identified out of the 10 tags present. This calculation is based on the analysis of framed Aloha with no capture.

In the next subsection, we explain why the detection rate for cards organized in a single stack and cards held in the player's hand is much lower than one would expect from the tag collision analysis.

\subsection{Tag detuning}

In inductively coupled RFID systems the voltage induced in the antenna coil of the tag by the magnetic field is used to power the microchip. Finkenzeller [2] describes how tag manufacturers create a parallel resonance circuit by adding a capacitor in parallel to the antenna coil so that the resonance frequency of the resonance circuit is tuned to the operating frequency of the RFID system. At resonance, the induced voltage produced across the tuned tag will thus be significantly enhanced compared to frequencies outside the resonant bandwidth resulting in an increased read range.

As a resonant application, the tag is, however, vulnerable to environmental detuning effects which can also cause a significant reduction in reading distance. Clusters of RFID tags in close proximity to each other, for example, exhibit significant detuning effects caused by their mutual inductances. Undesirable changes in the tag's parasitic capacitance and effective inductance can also be caused by metal and different dielectric mediums in the vicinity, e.g. a hand holding the tag [2]. The shift in resonance frequency away from the operating frequency results in the tag receiving less energy from the reader field and hence a decrease in reading distance.

Tag detuning due to other tags in very close proximity is thus also the cause for the low read rates we witnessed for the cards organized in a stack (see Figure 2 for details). The relatively low read rate in the player's hand is believed to be a result of the combination of tag detuning by the player's hand, by the proximity of the tags, and due to the increased distance from the antenna. 


\subsection{Other sources of error}

Other causes of failed reads include the presence of metal in the tag vicinity, since it distorts the magnetic flux, thus weakening the energy coupling to the tag. If tags are directly attached to a metal surface, they can often not be detected at all. Similar to tag detuning, metal in the vicinity of the reader antenna results in a read range reduction because the antenna is detuned. For example, in our experiments we witnessed a strong read range variation when we placed the reader antenna on a table that was supported by a metal frame.

The misalignment of the tags with the magnetic field of the reader coil can also lead to failed reads. Maximum power transfer occurs when the tag coil plane is perpendicular to the magnetic field lines. As the label is rotated with respect to the field lines, the coupling is reduced until the tag is no longer detected.

\section{Implications for system and application design}

To reduce the uncertainty that arises from the tag collisions, one could opt for RFID systems in which the time it takes to detect the tags is reduced. Since regulations on the $915 \mathrm{MHz}$ band offer significantly more bandwidth in the communication from the reader to the tag than do the regulations on the $13.56 \mathrm{MHz}$ band, an RFID system operating in the UHF band can detect tags much faster. The large bandwidth under US regulations also permits the use of deterministic anti-collision algorithms such as variants of the binary tree-walking scheme, where the reader traverses a tree of all possible identification numbers [4]. Unfortunately, the large bandwidth in the UHF band is currently not available worldwide, although there are proposals to increase the allocated bandwidth in Europe [2]. There are also RFID systems that exhibit a superior performance at $13.56 \mathrm{MHz}$, but they rely on more expensive tag and reader designs, e.g. involving the use of multiple frequency channels for the tag to reader communication.

To reduce the tag detuning that occurs when the tags are placed in a stack, we experimented with smaller tags that we placed in random locations on the playing cards. This reduced the tag detuning significantly, but the read range was also decreased due to the smaller labels. Alternatively, there are also specialized tags and readers available that are tuned for stack reading. Redundant tags placed at different orientations on the object to be identified are especially effective at reducing failed reads caused by a misalignment of the tag with the magnetic field of the reader antenna, as at least one of the tags should always be correctly aligned.

From an application design perspective, it is possible to use certain applicationspecific constraints. This might include group constraints, where a certain group of tags is known to always move together. The presence of a tag that was not detected can now be inferred by the detection of another tag that belongs to the same group. This group constraint would be particularly useful in cases where low cost RFID tags are used in conjunction with tags that are less likely to be affected by the issues presented above, e.g. active tags that are battery powered. 
Alternatively, the use of additional identification means that augment the RFID system could be considered, e.g. computer vision techniques or weighing scales.

The most radical approach to improve the performance of an RFID supported application is to modify the application itself so that a minimum number of tags are present in the read range simultaneously and the tags which are present are far apart from each other. The tag antennas should also be aligned with the magnetic field of the reader antenna, and metal and water should ideally not be present in the vicinity of the system. Unfortunately, this approach usually requires the active involvement of the user, e.g. by telling him not to place the cards in a stack. It also significantly reduces the appeal of RFID technology as a non-obtrusive identification technology.

\section{Conclusion}

Radio Frequency Identification (RFID) technology is known to be well-suited to linking the physical and virtual world. Using playing cards augmented with RFID tags as an example, we highlighted some of the issues that arise when multiple tags are present in the read range simultaneously. The paper also addresses the implications of those weaknesses from an application and system design perspective.

\section{References}

1. Christian Decker, Uwe Kubach, and Michael Beigl. Revealing the retail black box by interaction sensing. In Proceedings of the ICDCS 2003, Providence, Rhode Island, 2003.

2. Klaus Finkenzeller. RFID Handbook: Radio-Frequency Identification Fundamentals and Applications. John Wiley \& Sons, 2000.

3. Kay Römer and Svetlana Domnitcheva. Smart playing cards: A ubiquitous computing game. Journal for Personal and Ubiquitous Computing (PUC), 6, 2002.

4. Sanjay E. Sarma, Stephen A. Weis, and Daniel W. Engels. RFID Systems and Security and Privacy Implications. In Workshop on Cryptographic Hardware and Embedded Systems, pages 454-470. Lecture Notes in Computer Science, 2002.

5. H. Vogt. Efficient object identification with passive RFID tags. In F. Mattern and M. Naghshineh, editors, International Conference on Pervasive Computing, volume 2414 of Lecture Notes in Computer Science, pages 98-113, Zurich, August 2002. Springer-Verlag.

6. D. Wan. Magic medicine cabinet: A situated portal for consumer healthcare. In Proceedings of the International Symposium on Handheld and Ubiquitous Computing, Karlsruhe, Germany, 1999.

7. R. Want, K.O. Fishkin, A. Gujar, and B.L. Harrison. Bridging physical and virtual worlds with electronic tags. In Proc. of ACM SIGCHI, pages 370-377, May 1999.

8. M. Weiser. The computer of the 21st century. Scientific American, pages 94-100, September 1991.

9. Jeffrey E. Wieselthier, Anthony Ephremides, and Larry A. Michaels. An exact analysis and performance evaluation of framed aloha with capture. IEEE Transactions on Communications, COM-37(2):125-137, 1989. 